\title{
Assessing the orbital selective Mott transition with variational wave functions
}

\author{
Luca F. Tocchio, Federico Arrigoni, Sandro Sorella, and Federico Becca \\ Democritos National Simulation Center, Istituto Officina dei Materiali del CNR, \\ and SISSA-International School for Advanced Studies, Via Bonomea 265, I-34136 Trieste, Italy
}

(Dated: July 11, 2021)

\begin{abstract}
We study the Mott metal-insulator transition in the two-band Hubbard model with different hopping amplitudes $t_{1}$ and $t_{2}$ for the two orbitals on the two-dimensional square lattice by using non-magnetic variational wave functions, similarly to what has been considered in the limit of infinite dimensions by dynamical mean-field theory. We work out the phase diagram at half filling (i.e., two electrons per site) as a function of $R=t_{2} / t_{1}$ and the on-site Coulomb repulsion $U$, for two values of the Hund's coupling $J=0$ and $J / U=0.1$. Our results are in good agreement with previous dynamical mean-field theory calculations, demonstrating that the non-magnetic phase diagram is only slightly modified from infinite to two spatial dimensions. Three phases are present: a metallic one, for small values of $U$, where both orbitals are itinerant; a Mott insulator, for large values of $U$, where both orbitals are localized because of the Coulomb repulsion; and the so-called orbitalselective Mott insulator (OSMI), for small values of $R$ and intermediate $U$ 's, where one orbital is localized while the other one is still itinerant. The effect of the Hund's coupling is two-fold: on one side, it favors the full Mott phase over the OSMI; on the other side, it stabilizes the OSMI at larger values of $R$.
\end{abstract}

PACS numbers: 71.27.+a, 71.10.Fd, 71.30.+h, 75.25.Dk

\section{INTRODUCTION}

The single-band Hubbard model represents the simplest example where the competition between kinetic energy and Coulomb repulsion gives rise to a complex phase diagram, which is still representing a formidable problem to be solved in the theory of strongly-correlated systems. In many respects, the single-band Hubbard model can be considered to describe materials with partially occupied $d$ or $f$ shells. Although this approximation may capture some important aspects of these systems, like for example the Mott metal-insulator transition (MIT) and, possibly, the existence of $d$-wave superconductivity when doping a Mott insulator, retaining a single band is not always sufficient to correctly capture the low-energy properties of materials. Indeed, there are many examples in which orbital fluctuations are important and give rise to new physical phenomena. ${ }^{112}$ They include cases where the Coulomb exchange, which generates the Hund's rules, the existence of crystal fields or Jahn-Teller effects, and bandwidth differences among the orbitals produce appreciable effects at low temperatures.

Based on these premises, there is a compelling need to go beyond the single-band Hubbard model in order to clarify the role of orbital degeneracy, inter-orbital Coulomb interaction, and Hund's coupling. In particular, the MIT in multi-orbital systems involves other energy scales, besides the on-site Coulomb repulsion $U$ and the electron bandwidth. In order to highlight the effect of different terms, many studies have been performed in the non-magnetic sector, namely "neglecting" any possible magnetic long-range order. The aim of this choice is to capture the physics that is driven solely by electronic correlation and that can be released when magnetic order is destroyed by the presence of frustration (e.g., competing super-exchange couplings). Addressing the properties in the non-magnetic sector can be easily considered by different approaches, such as dynamical mean-field theory (DMFT) ${ }^{3}$ or slave-boson $(\mathrm{SB})^{\sqrt{4}}$ techniques, but also within variational Monte Carlo (VMC) $\underline{5}$ For example, whenever magnetic phases are not taken into account, DMFT and SB calculations have suggested that the Hund's coupling $J$ has a different effect for different filling factors: at half filling, it reduces the value $U_{\text {MIT }}$ above which the Mott state is stabilized, while, for all the other (integer) fillings, the presence of a finite $J$ increases $U_{\text {MIT }}{ }^{6 / 7}$ Another aspect that induces interesting variations in the description of the MIT is the existence of different bandwidths for degenerate orbitals $\underline{8}$ In the past, this issue has been deeply investigated on two-band models on the square lattice with hoppings $t_{1}$ and $t_{2}$, both intra- and inter-orbital Coulomb repulsions, and possibly also the Hund's exchange terms, by means of DMFT ${ }^{[15}$ and SB or slave-particle approaches 11116 In this case, whenever the ratio $R=t_{2} / t_{1}$ is sufficiently small (assuming $R \leq 1$ ), the two orbitals have distinct MITs by increasing the Coulomb repulsion, which implies the existence of an intermediate phase where one orbital is insulating and the other one is metallic; this phase has been named orbital-selective Mott insulator (OSMI). Instead, when the two orbitals have comparable hopping amplitudes, i.e., for $R$ larger than a critical value, a single MIT is present, where both orbitals undergo a simultaneous transition. Recently, it has been proposed that an OSMI can be stabilized also when the orbitals have the same bandwidth, provided they have different band dis-

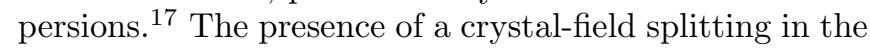
Hamiltonian is also responsible for the appearance of an OSMI $1 8 \longdiv { 2 0 }$

The possibility of a phase where some $d$ orbitals give rise to delocalized bands while some others re- 
main localized has been discussed in connection with $\mathrm{Ca}_{2-x} \mathrm{Sr}_{x} \mathrm{RuO}_{4}$, to explain the coexistence of spin- $1 / 2$ moments and metallicity at $x=0.5 .21 \sqrt[23]{23} \mathrm{~A}$ partial localization of $f$ electrons in some Uranium-based heavyfermion compounds has been also proposed to explain the observed Haas-van Alphen frequencies in $\mathrm{UPt}_{3} \cdot \frac{24}{2 n}$ this context, hopping anisotropies driven by intra-atomic correlations have been proposed as the driving mechanism for partial localization. ${ }^{25}$ Moreover, the orbital-selective Mott transition is conceptually equivalent to the Kondo breakdown in heavy-fermion systems, $\stackrel{26]}{,}$ where the localized $f$ electrons suddenly stop to hybridize with the conducting $c$ electrons and no longer contribute to the Fermi volume (which is determined by $c$ electrons only) ${ }^{27} \mathrm{In}$ this respect, a sign-problem-free model with one itinerant and one fully localized band has been studied by Determinant Monte Carlo.28

As mentioned, the issue of MITs in multi-orbital models with different hopping amplitudes has been investigated mainly by using DMFT, which is exact in infinite dimensions, and SB, which is a simple mean-field approximation; by contrast, very few attempts have been done with correlated methods that work in finite spatial dimensions. $\frac{29 \mid 30}{20}$ In this paper, we examine the phase diagram of the Hubbard model in two dimensions, with two degenerate orbitals and $R \leq 1$, by using correlated variational wave functions that are straightforward generalizations of the Jastrow-Slater states that have been widely used to study the single-band Hubbard model in the recent past. ${ }^{31132}$ In particular, the Jastrow factor is considered on top of an uncorrelated state, in order to correctly describe the effect of electron-electron interaction. Here, the uncorrelated determinant can be factorized into two terms for the different orbitals; the crucial ingredient is the inter-orbital Jastrow factor that couples densities on different orbitals and allows us a reliable determination of the various phases.

The outcomes of our variational approach are in good agreement with the ones that have been obtained by DMFT $10[1115$ This fact suggests that the (metastable) non-magnetic phase diagram of the model does not change much from infinite to two dimensions. It is also remarkable that relatively simple variational wave functions are able to capture most of the important physical properties also in cases where more than one orbital is involved, making it possible to use a similar technique also for other (more complicated) multi-orbital systems.

The paper is organized as follows: in Sec. II. we introduce the two-band Hubbard model and the variational wave functions that are used to study it; in Sec. III, we present the numerical results obtained by using variational Monte Carlo for $J=0$ and $J / U=0.1$; finally, in Sec. IV we draw our conclusions.

\section{MODEL AND METHOD}

We consider the two-band Hubbard model defined by:

$$
\mathcal{H}=\mathcal{H}_{\text {kin }}+\mathcal{H}_{\text {int }}
$$

where the kinetic term $\mathcal{H}_{\text {kin }}$ describes hopping processes of electrons within the two orbitals:

$$
\mathcal{H}_{\text {kin }}=-\sum_{\langle i, j\rangle, \alpha, \sigma} t_{\alpha} c_{i, \alpha, \sigma}^{\dagger} c_{j, \alpha, \sigma}+\text { h.c. }
$$

where $c_{i, \alpha, \sigma}^{\dagger}\left(c_{i, \alpha, \sigma}\right)$ creates (destroys) an electron with spin $\sigma$ on site $i$ and orbital $\alpha=1,2$ and $t_{\alpha}$ is the nearestneighbor hopping amplitude with orbital index $\alpha$. We define $R=t_{2} / t_{1}$ as the ratio between the two hopping parameters and, without loss of generality, we focus on the case with $R \leq 1$. In the following, we also fix $t_{1}=1$. We would like to stress the fact that the kinetic term is diagonal in the orbital index and, therefore, there is no a direct hybridization between different orbitals.

The interaction term includes different contributions:

$$
\begin{aligned}
\mathcal{H}_{\mathrm{int}} & =U \sum_{i, \alpha} n_{i, \alpha, \uparrow} n_{i, \alpha, \downarrow}+U^{\prime} \sum_{i, \sigma, \sigma^{\prime}} n_{i, 1, \sigma} n_{i, 2, \sigma^{\prime}} \\
& -J \sum_{i, \sigma, \sigma^{\prime}} c_{i, 1, \sigma}^{\dagger} c_{i, 1, \sigma^{\prime}} c_{i, 2, \sigma^{\prime}}^{\dagger} c_{i, 2, \sigma} \\
& -J^{\prime} \sum_{i}\left(c_{i, 1, \uparrow}^{\dagger} c_{i, 1, \downarrow}^{\dagger} c_{i, 2, \uparrow} c_{i, 2, \downarrow}+\text { h.c. }\right),
\end{aligned}
$$

where $n_{i, \alpha, \sigma}=c_{i, \alpha, \sigma}^{\dagger} c_{i, \alpha, \sigma}$ is the electronic density per spin on site $i$ and orbital $\alpha$. These four terms represent the intra-orbital interaction $U$, the inter-orbital interaction $U^{\prime}$, the Hund's coupling $J$, and the pair hopping $J^{\prime}$.

In order to study the occurrence of the OSMI, we focus on the half-filled case, i.e., two electrons per site. There are 6 atomic states with 2 particles per site. In particular, the states that diagonalize the single-site Hamiltonian are one (three-fold degenerate) triplet with energy $E=$ $U^{\prime}-J$ :

$$
\begin{aligned}
|1\rangle & =c_{i, 1, \uparrow}^{\dagger} c_{i, 2, \uparrow}^{\dagger}|0\rangle, \\
|2\rangle & =c_{i, 1, \downarrow}^{\dagger} c_{i, 2, \downarrow}^{\dagger}|0\rangle, \\
|3\rangle & =\frac{1}{\sqrt{2}}\left(c_{i, 1, \uparrow}^{\dagger} c_{i, 2, \downarrow}^{\dagger}-c_{i, 2, \uparrow}^{\dagger} c_{i, 1, \downarrow}^{\dagger}\right)|0\rangle,
\end{aligned}
$$

one singlet with electrons on different orbitals and energy $E=U^{\prime}+J:$

$$
|4\rangle=\frac{1}{\sqrt{2}}\left(c_{i, 1, \uparrow}^{\dagger} c_{i, 2, \downarrow}^{\dagger}+c_{i, 2, \uparrow}^{\dagger} c_{i, 1, \downarrow}^{\dagger}\right)|0\rangle,
$$

and, finally, two singlets with electrons on the same orbital and energies $E=U \pm J^{\prime}$ :

$$
\begin{aligned}
& |5\rangle=\frac{1}{\sqrt{2}}\left(c_{i, 1, \uparrow}^{\dagger} c_{i, 1, \downarrow}^{\dagger}-c_{i, 2, \uparrow}^{\dagger} c_{i, 2, \downarrow}^{\dagger}\right)|0\rangle, \\
& |6\rangle=\frac{1}{\sqrt{2}}\left(c_{i, 1, \uparrow}^{\dagger} c_{i, 1, \downarrow}^{\dagger}+c_{i, 2, \uparrow}^{\dagger} c_{i, 2, \downarrow}^{\dagger}\right)|0\rangle .
\end{aligned}
$$


According to the rotational symmetry of degenerate orbitals, we set $U^{\prime}=U-2 J$ and $J^{\prime}=J .33134$ In this case, for $J=0$ the ground state of the single site is sixfold degenerate, with $E=U$; in the most general case with $J>0$ instead, the ground state is the triplet with $E=U-3 J$, separated by a doubly-degenerate singlet (i.e., $|4\rangle$ and $|5\rangle$ ) with $E=U-J$; finally the singlet $|6\rangle$ has the highest energy $E=U+J$.

Our numerical results are based on the definition of variational wave functions that approximate the ground-state properties beyond perturbative approaches. We consider non-magnetic states as described by the Jastrow-Slater wave function that extends the original formulation by Gutzwiller.5135

$$
|\Psi\rangle=\mathcal{J}\left|\Phi_{0}\right\rangle
$$

where $\left|\Phi_{0}\right\rangle$ is an uncorrelated state that corresponds to the ground state of a BCS Hamiltonian:

$$
\begin{aligned}
& \mathcal{H}_{\mathrm{BCS}}=\sum_{k, \alpha, \sigma} \xi_{k}^{\alpha} c_{k, \alpha, \sigma}^{\dagger} c_{k, \alpha, \sigma} \\
& +\sum_{i, \sigma} \tilde{t}_{\perp}\left(c_{i, 1, \sigma}^{\dagger} c_{i, 2, \sigma}+c_{i, 2, \sigma}^{\dagger} c_{i, 1, \sigma}\right) \\
& +\sum_{k, \alpha} \Delta_{k}^{\alpha}\left(c_{k, \alpha, \uparrow}^{\dagger} \uparrow_{-k, \alpha, \downarrow}^{\dagger}+c_{-k, \alpha, \downarrow} c_{k, \alpha, \uparrow}\right) \\
& +\sum_{i}\left[\left(\Delta_{\perp}^{s}+\Delta_{\perp}^{t}\right) c_{i, 1, \uparrow}^{\dagger} c_{i, 2, \downarrow}^{\dagger}+\left(\Delta_{\perp}^{s}-\Delta_{\perp}^{t}\right) c_{i, 2, \uparrow}^{\dagger} c_{i, 1, \downarrow}^{\dagger}\right. \\
& \left.+\left(\Delta_{\perp}^{s}+\Delta_{\perp}^{t}\right) c_{i, 2, \downarrow} c_{i, 1, \uparrow}+\left(\Delta_{\perp}^{s}-\Delta_{\perp}^{t}\right) c_{i, 1, \downarrow} c_{i, 2, \uparrow}\right] .(11)
\end{aligned}
$$

The free intra-orbital dispersions $\xi_{k}^{\alpha}$ are given by:

$$
\xi_{k}^{\alpha}=-2 \tilde{t}_{\alpha}\left[\cos k_{x}+\cos k_{y}\right]-\mu_{\alpha},
$$

$\tilde{t}_{2}, \mu_{1}$, and $\mu_{2}$ being variational parameters that are optimized to minimize the variational energy (while $\tilde{t}_{1}=1$ sets the energy scale of the BCS Hamiltonian). Even though we allow for the presence of a further inter-orbital hopping $\tilde{t}_{\perp}$ in the BCS Hamiltonian (11), we find that the optimal variational states are obtained taking $\tilde{t}_{\perp}=0$. The best intra-orbital pairing terms have $d$-wave symmetry:

$$
\Delta_{k}^{\alpha}=2 \Delta^{\alpha}\left[\cos k_{x}-\cos k_{y}\right]
$$

with $\Delta_{k}^{\alpha}$ being further variational parameters, in analogy with the one-band Hubbard model for the square lattice. ${ }^{36 / 37}$ In addition, inter-orbital terms are considered, either with singlet (i.e., $\Delta_{\perp}^{s}$ ) or with triplet (i.e., $\left.\Delta_{\perp}^{t}\right)$ symmetries. As a result, we find that the interorbital singlet component is never relevant in the phase diagram, while the triplet component is necessary to correctly describe the full Mott phase in the presence of a finite Hund's coupling $J$, as pointed out also by the Gutzwiller approximation.

The effects of correlations are introduced by means of the so-called Jastrow factor $\mathcal{J}$ :

$$
\mathcal{J}=\exp \left(-\frac{1}{2} \sum_{i, j, \alpha, \beta} v_{i, j}^{\alpha, \beta} n_{i, \alpha} n_{j, \beta}\right),
$$

where $n_{i, \alpha}=\sum_{\sigma} n_{i, \alpha, \sigma}$ is the electron density on site $i$ and orbital $\alpha ; v_{i, j}^{\alpha, \beta}=v_{i, j}^{\beta, \alpha}$ (that include also the local Gutzwiller term for $\alpha=\beta$ and $i=j$ ) are pseudopotentials that are optimized for every independent distance $\left|\mathbf{R}_{i}-\mathbf{R}_{j}\right|$. The Jastrow factor has been shown to be crucial in describing a Mott insulating state within the single-band Hubbard model, with $v_{q} \propto 1 / q^{2}\left(v_{q}\right.$ being the Fourier transform of $v_{i, j}$ ) in the insulating region, while $v_{q} \propto 1 / q$ is found in the metallic/superconducting phase $\frac{3132}{132}$ We remark that the Jastrow factor embodies a crucial long-range attraction between doubly occupied and empty sites, keeping them bounded in the Mott phase 3113941 Otherwise, a non-magnetic Mott insulator cannot be obtained by using only a local (i.e., on-site) Gutzwiller term ${ }^{5}$ Similar results have been obtained in the bilayer Hubbard model ${ }^{42}$ Also in this paper on the two-band Hubbard model, we show that the presence of a Jastrow factor is necessary to obtain a non-trivial phase diagram with metallic and Mott phases, as well as the OSMI. We remark that, while an insulating state can be obtained just by applying the Jastrow factor on top of a Fermi gas, the pairing terms of the BCS Hamiltonian are particularly important to have a correct description of Mott insulators, where localized electrons are paired together to form the RVB state, as proposed originally by Anderson. 43

We mention that the Jastrow-Slater wave functions of Eq. 10. may be improved by considering the so-called backflow correlations, ${ }^{44 / 45}$ which are particularly important at half filling and for small hole dopings; however, the physical content of the original variational states is not modified by the inclusion of backflow terms. Therefore, in the following we will not consider these corrections, which are relatively computationally expensive.

The wave function obtained from applying the Jastrow factor to the ground state of the BCS Hamiltonian of Eq. (11) does not describe phases with magnetic longrange order; also phases with orbital order cannot be captured. In this sense, as discussed in the introduction, our variational states are suitable to approach the paramagnetic Mott transition, driven solely by electronic correlations. We would like to mention the fact that orbital order may be obtained whenever, in the BCS Hamiltonian (11), the $d$-wave intra-orbital pairing is replaced by an on-site $s$-wave one:

$$
\sum_{k}\left[\Delta_{1} c_{k, 1, \uparrow}^{\dagger} c_{-k, 1, \downarrow}^{\dagger}+\Delta_{2} c_{k, 2, \uparrow}^{\dagger} c_{-k, 2, \downarrow}^{\dagger}+\text { h.c. }\right] .
$$

Indeed, this pairing term gives a sizable energy gain for $J=0$, due to the appearance of a staggered orbital order, where the orbital 1 is (almost) doubly occupied and the orbital 2 is (almost) empty on one sublattice and viceversa for the other sublattice. Remarkably, although the BCS Hamiltonian of Eq. 15 is translationally invariant (implying a translationally invariant $\left|\Phi_{0}\right\rangle$ ), densitydensity correlations computed with the wave function $|\Psi\rangle$ of Eq. 10 clearly show long-range orbital order. The fact that (correlated) translationally invariant wave 


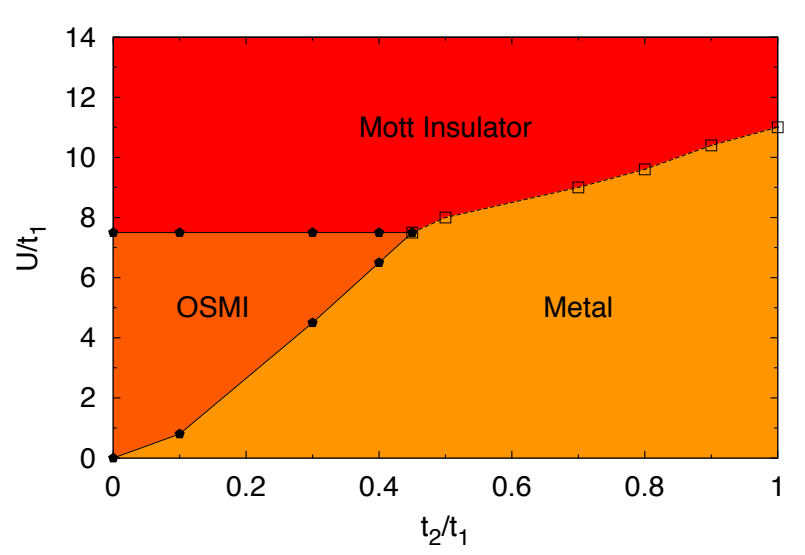

FIG. 1: (Color online) Non-magnetic phase diagram of the two-band Hubbard model with $J=0$. Three regions can be identified as a function of $R$ and $U / t_{1}$ : a metal (where both orbitals are metallic), a full Mott insulator (where both orbitals are insulating), and the orbital-selective Mott insulator (where the orbital with the smallest bandwidth is insulating while the one with the largest bandwidth is metallic). Continuous lines denote second-order transitions, while the dashed line denotes a first-order transition.

functions may show long-range order has been already discussed in one-band models, where dimerization ${ }^{31}$ or charge $\operatorname{order}^{[46}$ can be obtained, and has been investigated in detail in Ref. 47 .

Finally, we would like to emphasize the advantages and disadvantages of the variational Monte Carlo method. The main advantage is that correlated states may be considered and treated beyond any perturbative approach and without any approximation (e.g., without the Gutzwiller approximation $\frac{48 / 49}{}$ ). However, in order to compute expectation values over variational states, a Monte Carlo sampling is necessary, thus leading to statistical errors. The energy computed with variational Monte Carlo gives an upper bound to the exact value, thus providing a criterion to judge the quality of the variational states. Moreover, it is possible to assess quite large clusters, with all relevant spatial symmetries (translations, rotations, and reflections) preserved. By contrast, it is difficult to quantify the systematic errors, which are introduced by the choice of the trial state.

\section{RESULTS}

In this section, we present the variational results obtained by using the Jastrow-Slater wave function of Eq. (10). We study the model on two-dimensional square lattices with $L$ sites and take 45 -degree tilted clusters with $L=2 l^{2}$ sites, $l$ being an odd integer. First, we consider the case with $J=0$, then we study the effect of a small Hund's coupling, i.e., $J / U=0.1$.
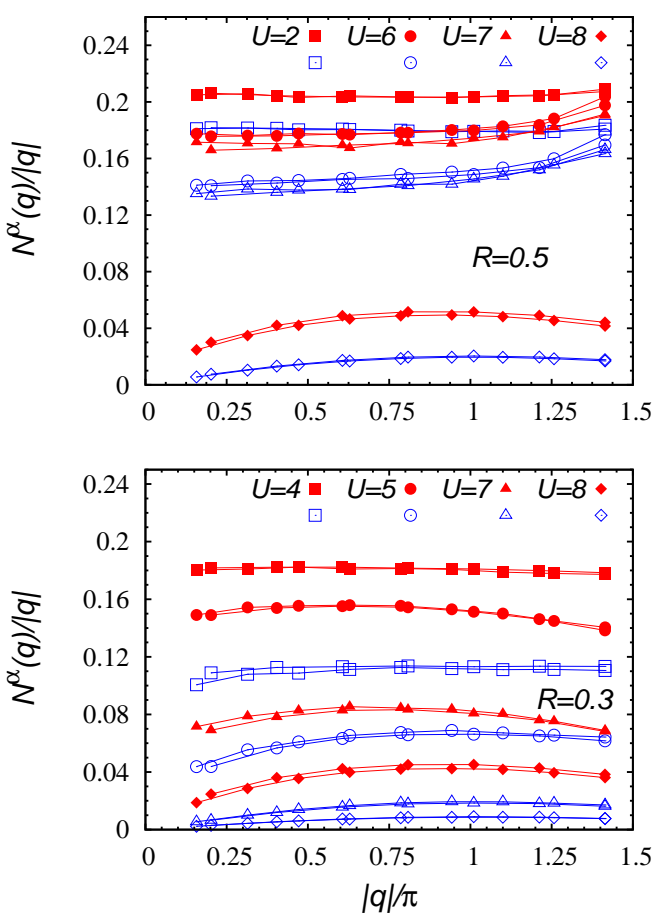

FIG. 2: (Color online) Intra-orbital density-density structure factor $N^{\alpha}(q)$ divided by $|q|$ as a function of $|q| / \pi$ along the $\Gamma-X$ line, where $\Gamma=(0,0)$ and $X=(\pi, \pi)$ in the first Brillouin zone. Data are shown for different values of $U / t_{1}$ at $R=0.3$ and at $R=0.5$ for $J=0$. Red full symbols refer to the case $\alpha=1$, while empty blue symbols refer to $\alpha=2$. Results are shown for $L=98$ and 162. Statistical error bars are smaller than the symbol size.

\section{A. The case with $J=0$}

Let us start by pointing out that, if no inter-orbital coupling is present in the Hamiltonian of Eq. (3) (i.e., $U^{\prime}=0$ and $J=J^{\prime}=0$ ), the OSMI would take place in a quite large region of the phase diagram. Indeed, the full Hamiltonian (1) would decouple into two singleband Hubbard models, with the same Coulomb repulsion $U$ but different hopping amplitudes, e.g., $R<1$. In the non-magnetic sector, the two orbitals would have distinct MITs, because $R \neq 1$. The phase diagram, in the $\left(R, U / t_{1}\right)$ plane would be very simple: i) a Mott phase for $U>U_{\mathrm{MIT}}$, where $U_{\mathrm{MIT}}$ is the critical value for the single-band model; ii) a metallic phase for $U<U_{\mathrm{MIT}} R$; and iii) an OSMI for $U_{\mathrm{MIT}} R<U<U_{\mathrm{MIT}}$. These (trivial) results are obtained within the variational wave function 10 by imposing a vanishing inter-orbital Jastrow factor in Eq. 14 , i.e., $v_{i, j}^{\alpha, \beta}=0$ for $\alpha \neq \beta$. In this case, $U_{\mathrm{MIT}} / t_{1}=7.5 \pm 0.5$ [50

The results are substantially modified in the presence of the inter-orbital coupling $U^{\prime}=U(J=0)$, which favors the metallic phase over a much larger region. Within the variational approach, this effect is captured by allowing an inter-orbital Jastrow factor $v_{i, j}^{\alpha, \beta}$ with $\alpha \neq \beta$ in 
Eq. (14). Our results, obtained from calculations on 98 and 162 sites (with two orbitals per site) are summarized in Fig. 1, where we report the ground-state phase diagram in the $\left(R, U / t_{1}\right)$ plane. We notice that, as long as the value of $R$ is sufficiently small, e.g., $R \lesssim 0.5$, the two orbitals stay essentially decoupled, and the OSMI may exist at intermediate Coulomb interactions. In addition, the critical $U$ leading to the full Mott phase does not depend upon $R$, as expected, since the two orbitals are decoupled. By contrast, for $R \gtrsim 0.5$, the OSMI disappears, given the effective hybridization between the two orbitals. Here, the value of $U_{\mathrm{MIT}}$, at which the Mott state takes place, increases monotonically with $R$. This result is consistent with what has been suggested by a Monte Carlo study of the degenerate $M$-band Hubbard model on the square lattice with $R=1$, where $U_{\mathrm{MIT}} / W \simeq \sqrt{M}$ ( $W=8 t_{1}$ being the bandwidth) ${ }^{51}$ For $M=2$ (and $R=1$ ), a larger value for the MIT, i.e., $U_{\mathrm{MIT}} / W \simeq 2$, has been instead predicted by the slave-boson approach of Ref. ${ }^{[2]}$. Our present results for the whole phase diagram are qualitatively similar to what has been obtained within DMFT by several authors in the past 1011115$]$ Some quantitative discrepancies may be observed in the location of the transition to the full Mott insulator, that is located at slightly higher values of $U / t_{1}$ in our approach. Moreover, we find a larger region of OSMI, that extends to $R \approx 0.45$ instead of $R \approx 0.2$, as obtained in DMFT.

We remark that the OSMI is stable also when including a direct (on-site) inter-orbital hopping $\tilde{t}_{\perp}$ in the meanfield Hamiltonian of Eq. (11). Indeed, even if this possibility is allowed, the optimal variational state has $\tilde{t}_{\perp}=0$. In addition, we also verified that each orbital remains half-filled even if charge transfer processes are allowed within the Monte Carlo moves.

In practice, the metallic or insulating nature can be determined by looking at the static density-density structure factor ${ }^{3132 / 45}$ Let us briefly discuss this issue on the single-band Hubbard model and then generalize it to the two-band case. Consider:

$$
N(q)=\frac{\left\langle\Psi\left|n_{-q} n_{q}\right| \Psi\right\rangle}{\langle\Psi \mid \Psi\rangle}
$$

where $n_{q}=1 / \sqrt{L} \sum_{r} e^{i q r} n_{r}$ is the Fourier transform of the particle density $n_{r}$ (on a single-band model there is only one orbital and we drop the index for that). A metallic behavior is characterized by $N(q) \propto|q|$ for $q \rightarrow 0$, which implies a vanishing gap for particle-hole excitations. On the contrary, $N(q) \propto q^{2}$ for $q \rightarrow 0 \mathrm{im}-$ plies a finite gap and an insulating behavior. These facts are a consequence of approximating the lowest-energy excitations by the Feynman construction (the so-called single-mode approximation) as $\left|\Psi_{q}\right\rangle=n_{q}|\Psi\rangle$ !53

Here, we are considering a model where there is no direct hybridization between the two orbitals in the $J=0$ case and where the charge transfer between the orbitals remains negligible also in the presence of the pair hopping term in the Hamiltonian. Therefore, since we are interested in the properties of each orbital individually,
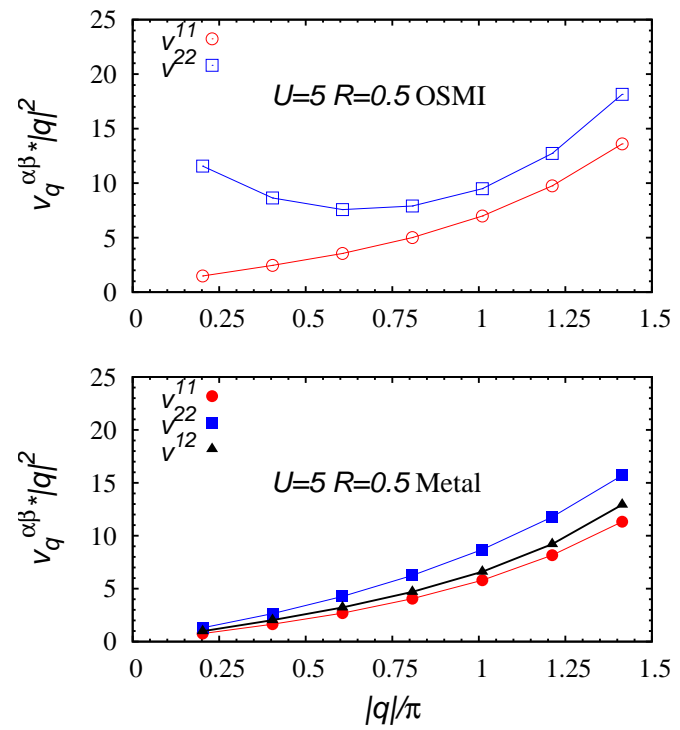

FIG. 3: (Color online) The case with $U / t_{1}=5$ and $R=0.5$, which falls in the OSMI if the two orbitals are not interacting, i.e., $U^{\prime}=0$ and $J=0$, while it falls in the metallic phase for $U^{\prime}=U(J=0)$. Upper panel: intra-orbital Jastrow factors $v_{q}^{\alpha, \alpha}$ multiplied by $|q|^{2}$, as a function of $|q| / \pi$, for the case where a variational state is taken with $v_{i, j}^{1,2}=0$. In this case the $\alpha=1$ orbital is metallic (i.e., $v_{q}^{1,1} \propto 1 /|q|$ ), while the $\alpha=2$ orbital is insulating (i.e., $\left.v_{q}^{2,2} \propto 1 / q^{2}\right)$. Lower panel: Jastrow factors $v_{q}^{\alpha, \beta}$ multiplied by $|q|^{2}$, as a function of $|q| / \pi$, for the case where all Jastrow terms are optimized together. Here, the presence of the inter-orbital Jastrow factor stabilizes a metallic state, with $v_{q}^{\alpha, \alpha} \propto 1 /|q|$. In both panels, the $q$ points are along the $\Gamma-X$ line, with $\Gamma=(0,0)$ and $X=(\pi, \pi)$. The results are obtained for $L=98$. Statistical error bars are smaller than the symbol size.

we consider only the intra-orbital correlations:

$$
N^{\alpha}(q)=\frac{\left\langle\Psi\left|n_{\alpha,-q} n_{\alpha, q}\right| \Psi\right\rangle}{\langle\Psi \mid \Psi\rangle},
$$

where now $n_{\alpha, q}$ is the Fourier transform of the particle density on the orbital $\alpha$. The metallic or insulating behavior of the two orbitals can be assessed by looking at the small- $q$ behavior of $N^{\alpha}(q)$. The metallic (Mott) phase is characterized by $N^{\alpha}(q) \propto|q|\left(N^{\alpha}(q) \propto q^{2}\right)$ for both $\alpha=1$ and 2, while the OSMI has $N^{1}(q) \propto|q|$ and $N^{2}(q) \propto q^{2}$.

In Fig. 2 data are shown for the two cases $R=0.3$ and $R=0.5$. While in the first case an intermediate phase, with one orbital that is metallic and the other one that is insulating, can be identified as a function of $U / t_{1}$, in the second one, a direct transition between a fully metallic phase and a full Mott state is observed. Moreover, in the first case with $R=0.3$ the coefficient of the linear term in $N^{\alpha}(q)$ goes to zero smoothly when approaching the metal-insulator transition (for both bands). This result suggests that both the transition between the metallic and the OSMI phases and the one between the OSMI 

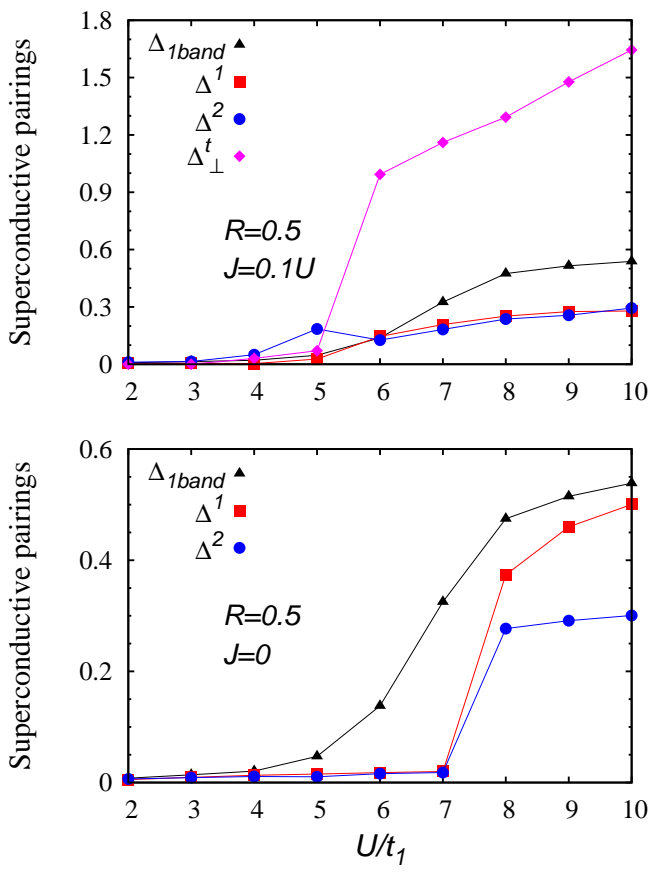

FIG. 4: (Color online) Superconducting pairing fields in the variational state as a function of $U / t_{1}$ for $R=0.5$ with $J / U=0.1$ (upper panel) and $J=0$ (lower panel). $\Delta^{1}$ and $\Delta^{2}$ represent intra-orbital $d$-wave pairing in the orbital 1 and 2 , respectively; $\Delta_{\perp}^{t}$ represents triplet pairing between different orbitals on the same site (shown only for $J / U=0.1$ ). The $d$-wave pairing field of the one-band case $\Delta_{1 \text { band }}$ is also shown for comparison. Data are presented for a $L=98$ lattice size. Statistical error bars are smaller than the symbol size.

and the Mott state are second order. This statement is further supported by the fact that no fully metallic state can be stabilized within the OSMI phase (and no OSMI can be stabilized within the Mott phase), as a local minimum. By contrast, for $R=0.5$ the coefficient of the linear term in both $N^{1}(q)$ and $N^{2}(q)$ suddenly drops at the transition. In addition, metastable solutions can be found, which indicates that the metal-insulator transition is first order.

The large metallic region that is observed in the phase diagram can be stabilized by the presence of the interorbital Jastrow factor. Indeed, a variational wave function without this term would show a much more extended region of OSMI, with a significantly higher variational energy. For example, for $U / t_{1}=5$ and $R=0.5$ the wave function without inter-orbital Jastrow factor has an energy that is $0.1 t_{1}$ higher than the best metallic solution with all Jastrow factors. In Fig. 3, we report the calculations for the Jastrow parameters in these two cases. We show that the presence of an inter-orbital Jastrow factor $v_{i, j}^{1,2}$ in the variational state is able to change the small- $q$ behavior of the intra-orbital Jastrow factor for the most correlated band, namely $v_{q}^{2,2}$, thus leading to a metallic behavior in both bands.

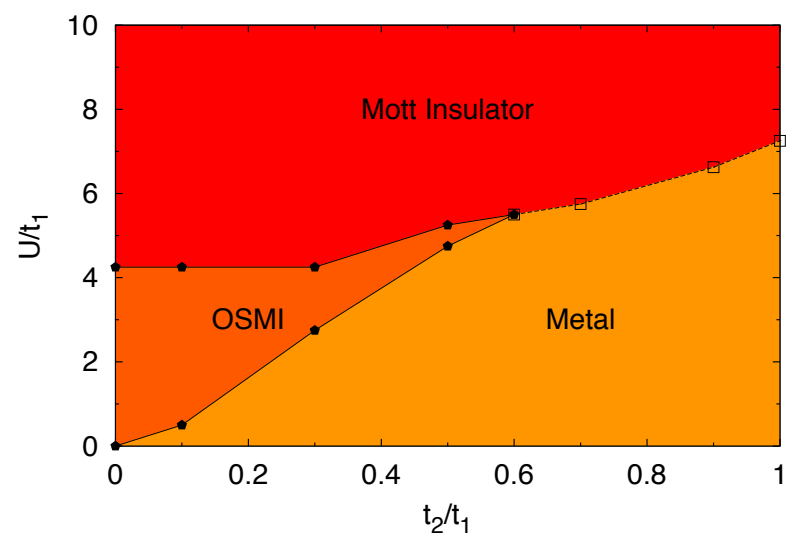

FIG. 5: (Color online) The same as in Fig. 1 but with $J / U=$ 0.1 .
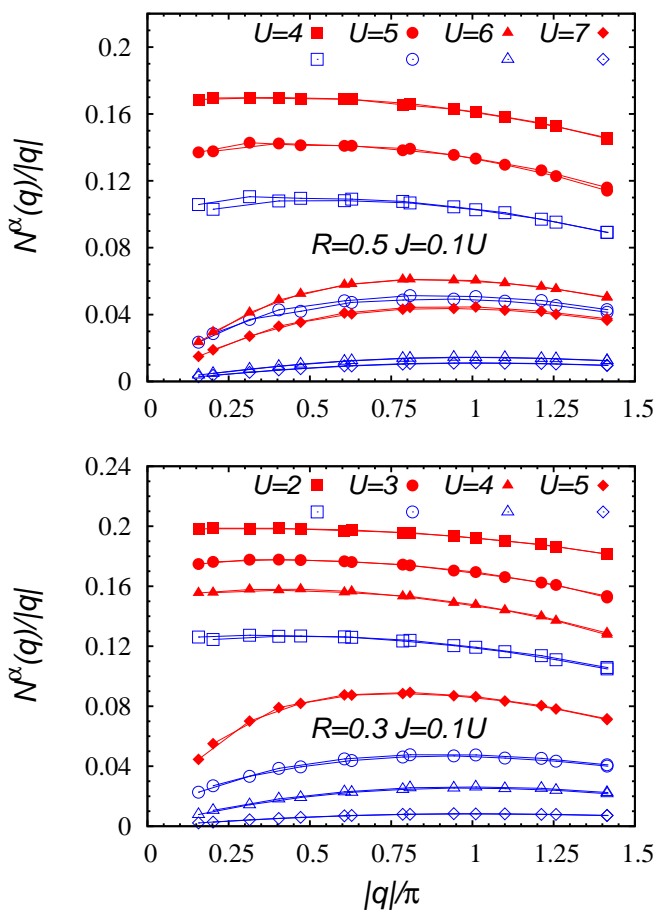

FIG. 6: (Color online) The same as in Fig. 2 but with $J / U=$ 0.1 .

Concerning the mean-field part of the variational state, we show in Fig. 4 (lower panel) the behavior of the intraorbital BCS pairings $\Delta^{1}$ and $\Delta^{2}$ of Eq. 13 , as a function of $U / t_{1}$, for a value of $R$ where a direct transition between a metallic and the full Mott state occurs. We notice that both the intra-orbital pairings have a jump at the transition, from a vanishingly small value in the metallic phase to a finite value when the bands are insulating. This behavior is different from what happens in the oneband Hubbard model, in which the BCS pairing increases smoothly with $U$, indicating a second-order transition in the thermodynamic limit. 
We finally remark that an orbital ordered state can be obtained by allowing for an on-site $s$-wave pairing field, as described in Eq. (15). Indeed, the presence of this term leads to a considerable energy gain and induces long-range orbital order with $n_{i, \alpha} \propto\left[1+(-1)^{R_{i}+\alpha}\right]$. The presence of orbital order can be detected by looking at the intra-orbital density-density correlations $N^{\alpha}(q)$. Indeed, the characteristic density pattern within each orbital, with doubly-occupied sites surrounded by empty sites, is reflected into a divergent peak of $N^{\alpha}(q)$ at the vector $Q=(\pi, \pi)$ (not shown). Even if we have evidence that orbital order would occur for any value of $U$ and $R$, a precise size scaling of $N^{\alpha}(Q)$ for small values of $U$ would require larger lattice sizes and is out of the scope of the present paper (that focuses on the paramagnetic Mott transition).

\section{B. The case with $J / U=0.1$}

Let us now turn to the case with a finite Hund's coupling $J$ and consider the case with $J / U=0.1$. The ground-state phase diagram in the $\left(R, U / t_{1}\right)$ plane is reported in Fig. 5, to be compared with the one for $J=0$ in Fig. 1. Moreover, in Fig. 6, we report the results for the density-density correlations of Eq. (17) for $R=0.3$ and $R=0.5$, to be compared with the case for $J=0$ in Fig. 2 Two remarks can be drawn. i) The presence of a finite $J$ term favors the full Mott state over both the OSMI and the metallic phase. As a result, the transition line that marks the stabilization of the Mott phase shifts down to lower values of $U / t_{1}$. This outcome can be easily understood from the fact that the Mott state, where all electrons are localized, has a large energy gain coming from the Hund's rule, which favors a spin alignment. ii) A finite $J$ coupling also favors the OSMI with respect to the metallic phase (i.e., the OSMI can be stabilized for larger values of $R$, up to 0.6 , with respect to the $J=0$ case). Within DMFT, this fact has been explained by a non-vanishing magnetic moment in the metallic phase when $J>0,{ }^{7}$ which may gain energy when coupled together with the one present in the insulating orbital. Moreover, we have that the critical $U$ that leads to the Mott phase for small $R$ is no longer independent from $R$ : here, $J$ directly couples the two orbitals and the transition point changes from $U_{\mathrm{MIT}} / t_{1}=4 \pm 0.5$ for $R \approx 0$ to $U_{\mathrm{MIT}} / t_{1}=5.5 \pm 0.5$ for $R=0.6$. This feature is somehow missing in the DMFT picture where the transition to the Mott phase is almost constant at $U_{\mathrm{MIT}} / t_{1} \sim 4.15$. We also remark that the critical $U$ predicted by our Monte Carlo approach for the MIT at $R=1$ is smaller than the slave-boson result, where $U / W \simeq 1.3 !^{[52}$

One important aspect is that a remarkable energy gain in the Mott phase is obtained by considering an on-site and inter-orbital triplet pairing $\Delta_{\perp}^{t}$ in the mean-field Hamiltonian (11). This outcome is natural, given the fact that for $J>0$, the atomic ground state of Eq. (3) is given by the triplet states of Eqs. (4), (5), and (6).
In Fig. 4 (upper panel) we report the BCS pairings as a function of $U / t_{1}$ at $R=0.5$. Three different regimes can be distinguished by increasing the Coulomb repulsion: a metallic phase for $U / t_{1} \leq 4$ where all the pairings are negligible, an OSMI phase at $U / t_{1} \approx 5$, where the largest pairing is the intra-orbital one on the most correlated band, and the Mott insulator, where in addition to the two $d$-wave intra-orbital pairings there is a large triplet pairing between different orbitals on the same site. This latter term encodes the ferromagnetic Hund's coupling part of the Hamiltonian. We finally remark that, in contrast to the $J=0$ case, no orbital order is observed for $J / U=0.1$, since this ordered state would be incompatible with the Hund's coupling, which favors triplet states.

\section{CONCLUSIONS}

In this paper, we examined a two-band Hubbard model in the case where the two orbitals have different hopping amplitudes, with particular emphasis on the existence of the orbital selective Mott insulator, that emerges in the non-magnetic sector. In the recent past, this topic has been widely addressed by mean-field methods, including $\mathrm{SB}$ approaches and DMFT, which is exact in infinite dimensions. Here, we made use of an alternative approach, based on variational wave functions with Jastrow terms, in order to capture long-range spatial correlations in two dimensions, thus providing a complementary approach to DMFT. The first outcome of our study is that the nonmagnetic phase diagram does not qualitatively change when going from infinite to two spatial dimensions: we confirm the existence of the OSMI phase already for the $J=0$ case; in addition we verify that the Hund's coupling is favoring the full Mott phase over the OSMI and the OSMI over the metal. The second outcome is more technical and refers to the fact that relatively simple variational wave functions are able to capture the important physical properties of multi-band Hubbard models, with different kinds of interactions. In particular, we highlighted the role of the inter-band Jastrow factor in properly describing the orbital hybridization and the role of the triplet inter-orbital pairing in capturing the effect of the Hund's coupling.

Our variational states can be naturally extended to describe three- or even five-orbital models, which are suitable to describe electrons in partially occupied $d$ shells. These settings would allow, for example, to study transition-metal oxides of $3 d$ and $4 d$ series (such as Cuprates and Ruthenates), as well as the iron-based superconductors (Iron Pnictides and Chalcogenides) $\left[\frac{54}{}\right.$ Finally, with the further inclusion of spin-orbit coupling, a three-band model is also appropriate to study the unconventional physics of $5 d$ materials, such as Iridates $\underline{55156}$ 


\section{Acknowledgments}

We would like to thank M. Fabrizio, R. Valentí, C. Gros, L. de' Medici, and J. Mravlje for useful discussions.

1 M. Imada, A. Fujimori, and Y. Tokura 1998 Rev. Mod. Phys. 701039.

${ }^{2}$ Y. Tokura and N. Nagaosa 2000 Science 288462.

3 A. Georges, G. Kotliar, W. Krauth, and M.J. Rozenberg 1996 Rev. Mod. Phys. 6813.

${ }^{4}$ G. Kotliar and A.E. Ruckenstein 1986 Phys. Rev. Lett. 57 1362.

${ }^{5}$ H. Yokoyama and H. Shiba 1987 J. Phys. Soc. Jpn. 56 1490.

6 L. de' Medici, J. Mravlje, and A. Georges 2011 Phys. Rev. Lett. 107256401.

7 L. de' Medici 2011 Phys. Rev. B 83205112.

8 A. Liebsch 2003 Phys. Rev. Lett. 91226401.

9 A. Koga, N. Kawakami, T. M. Rice, and M. Sigrist 2004 Phys. Rev. Lett. 92216402.

10 M. Ferrero, F. Becca, M. Fabrizio, and M. Capone 2005 Phys. Rev. B $\mathbf{7 2} 205126$.

11 L. de' Medici, A. Georges, and S. Biermann 2005 Phys. Rev. B 72205124.

12 R. Arita and K. Held 2005 Phys. Rev. B 72201102.

13 A. Liebsch 2005 Phys. Rev. Lett. 95116402.

14 C. Knecht, N. Blümer, and P.G.J. van Dongen 2005 Phys. Rev. B 72081103.

15 K. Inaba and A. Koga 2006 Phys. Rev. B 73155106.

16 A. Rüegg, M. Indergand, S. Pilgram, and M. Sigrist 2005 Eur. Phys. J. B 4855.

17 Y.-Z. Zhang, H. Lee, H.-Q. Lin, C.-Q. Wu, H.O. Jeschke, and R. Valentí 2012 Phys. Rev. B 85035123.

18 P. Werner and A.J. Millis 2007 Phys. Rev. Lett. 99126405.

19 L. de' Medici, S.R. Hassan, M. Capone, and X. Dai 2009 Phys. Rev. Lett. 102126401.

20 Y. Song and L.-J. Zou 2009 Eur. Phys. J. B 7259.

21 V.I. Anisimov, I.A. Nekrasov, D.E. Kondakov, T.M. Rice, and M. Sigrist 2002 Eur. Phys. J. B 25191.

22 S. Nakatsuji, D. Hall, L. Balicas, Z. Fisk, K. Sugahara, M. Yoshioka, and Y. Maeno 2003 Phys. Rev. Lett. 90137202.

${ }^{23}$ For a recent discussion on different aspects of the orbitalselective Mott transition, see for example, Y. Yao, Y.-Z. Zhang, H. Lee, H.O. Jeschke, R. Valentí, H.-Q. Lin, and C.-Q. Wu 2013 Mod. Phys. Lett. B 271330015.

24 G. Zwicknagl, A.N. Yaresko, and P. Fulde 2002 Phys. Rev. $B 65081103(\mathrm{R})$.

25 D.V. Efremov, N. Hasselmann, E. Runge, P. Fulde, and G. Zwicknagl 2004 Phys. Rev. B 69115114.

26 A. Schröder, G. Aeppli, E. Bucher, R. Ramazashvili, and P. Coleman 1998 Phys. Rev. Lett. 80 5623; A. Schröder, G. Aeppli, R. Coldea, M. Adams, O. Stockert, H. v. Löhneysen, E. Bucher, R. Ramazashvili, and P. Coleman 2000 Nature (London) 407351.

27 For a review on the orbital-selective Mott transition and its relation to the Kondo breakdown, see M. Vojta $2010 \mathrm{~J}$. Low. Temp. Phys. 161203.

${ }^{28}$ K. Bouadim, G.G. Batrouni, and R.T. Scalettar 2009 Phys.
We acknowledge support from PRIN 2010 2010LLKJBX.
Rev. Lett. 102226402.

29 A. Koga, N. Kawakami, H. Yokoyama, and K. Kobayashi 2006 AIP Conf. Proc. 8501458.

30 Y. Takenaka and N. Kawakami 2012 J. Phys.: Conf. Ser. 400032099.

31 M. Capello, F. Becca, M. Fabrizio, S. Sorella, and E. Tosatti 2005 Phys. Rev. Lett. 94026406.

32 M. Capello, F. Becca, S. Yunoki, and S. Sorella 2006 Phys. Rev. B 73245116.

33 J. Kanamori 1963 Prog. Theor. Phys. 30275.

34 C. Castellani, C. R. Natoli, and J. Ranninger 1978 Phys. Rev. B 184945.

35 M.C. Gutzwiller 1963 Phys. Rev. Lett. 10159.

36 C. Gros 1988 Phys. Rev. B 38 931(R).

37 F.C. Zhang, C. Gros, T.M. Rice, and H. Shiba 1988 Supercond. Sci. Technol. 136.

38 M. Zegrodnik, J. Bünemann, and J. Spalek 2014 New J. Phys. 16033001.

39 M. Capello, F. Becca, M. Fabrizio, and S. Sorella 2007 Phys. Rev. Lett. 99056402.

40 H. Yokoyama, T. Miyagawa, and M. Ogata 2011 J. Phys. Soc. Jpn. 80084607.

41 T. Miyagawa and H. Yokoyama 2011 J. Phys. Soc. Jpn. 80 084705.

42 R. Rüger, L.F. Tocchio, R. Valentí, and C. Gros 2014 New. J. Phys. 16033010.

43 P.W. Anderson 1987 Science 2351196.

44 L.F. Tocchio, F. Becca, A. Parola, and S. Sorella 2008 Phys. Rev. B $\mathbf{7 8}$ 041101(R).

${ }^{45}$ L.F. Tocchio, F. Becca, and C. Gros 2011 Phys. Rev. B 83 195138.

46 L.F. Tocchio, C. Gros, X.-F. Zhang, and S. Eggert 2014 Phys. Rev. Lett. 113246405.

47 R. Kaneko, L.F. Tocchio, R. Valentí, F. Becca, and C. Gros 2015 arXiv:1510.08653

48 W.F. Brinkman and T.M. Rice 1970 Phys. Rev. B 24302.

49 D. Vollhardt 1984 Rev. Mod. Phys. 5699.

50 For the single-band Hubbard model the value of $U_{\text {MIT }} / t_{1}$ slightly changes when allowing $d$-wave pairing in the variational wave function. The values are $U_{\mathrm{MIT}} / t_{1}=8.5 \pm 0.5$ if we fix $\Delta=0, \frac{32}{22}$ and $U_{\mathrm{MIT}} / t_{1}=7.5 \pm 0.5$ when a finite electron pairing is allowed.

51 O. Gunnarsson, E. Koch, and R.M. Martin 1996 Phys. Rev. $B 54$ 11026(R).

52 A. Klejnberg and J. Spalek 1998 Phys. Rev. B 5712041.

53 R.P. Feynman 1954 Phys. Rev. 94262.

54 A. Georges, L. de' Medici, and J. Mravlje 2013 Annu. Rev. Condens. Matter Phys. 4137.

55 H. Watanabe, T. Shirakawa, and S. Yunoki 2013 Phys. Rev. Lett. 110027002.

56 T. Sato, T. Shirakawa, and S. Yunoki 2015 Phys. Rev. B 91125122. 